\title{
REVISIONES
}

\section{El interés de la etnografía escolar en la investigación educativa}

\author{
The interest of school ethnography in educative research \\ O interesse da etnografia escolar na investigação educativa
}

Carmen Álvarez

Universidad de Cantabria, a lvarezmc@unican.es, 942202029

\begin{abstract}
RESUMEN
Frente a los enfoques de investigación de índole positivista han ido surgiendo diversas perspectivas alternativas en investigación educativa. Una de ellas es la etnografía escolar, que se centra en el estudio de la cultura que se desarrolla en los centros y en las aulas. En este artículo se plantea el interés que tiene para los investigadores el empleo de este modelo de investigación y trata de responder interrogantes fundamentales de este modelo de investigación: qué entendemos por etnografía escolar, qué características tiene, qué papel juega el investigador, cuáles son los principales momentos, cómo conseguir que la investigación etnográfica sea válida y qué avances supone con respecto a otros modelos de investigación.
\end{abstract}

Palabras clave: etnografía escolar, investigación educativa, observación participante, cultura escolar.

\begin{abstract}
Various alternative perspectives in educational research have emerged in relation to positivist research approaches. One is the school ethnography, which focuses on the study of culture promoted in schools and classrooms. This article raises the interest of researchers in using this model for research and attempts to answer fundamental questions of this model of research: what do we mean by school ethnography; what features it has; what's the role of the researcher; what are the main moments; how to validate ethnographic research; and what is its progress when compared to other research models.
\end{abstract}

Key words: school ethnography, educational research, participant observation, school culture.

\section{RESUMO}

Diante dos enfoques investigativos de cunho positivista, surgiram várias possibilidades alternativas na pesquisa educacional. Uma delas é a etnografia escolar que focaliza o estudo da cultura promovida em escolas e salas de aula. Este artigo suscita o interesse de pesquisadores neste modelo investigativo e tenta responder às questões fundamentais para esse tipo de investigação: o que entendemos por etnografia da escola? Quais recursos ela tem? Qual é a tarefa do pesquisador? Quais são as suas principais etapas? Como torná-la legítima e quais são os seus progressos, comparando-a a outros modelos pesquisa?

Palavras chave: etnografia escolar, pesquisa educacional, observação participante, cultura escolar.

\section{1. ¿QUÉ ENTENDEMOS POR ETNOGRAFÍA ESCOLAR?}

El concepto de "etnografía escolar" es un término ampliamente utilizado, aunque no siempre con los mismos sentidos. Se suele definir como una etnografía realizada en el campo específico de la escuela (Fernández Enguita, 1985; Woods, 1987; Goetz y Lecompte, 1988; Torres, 1988; Martínez Rodríguez, 1990; Aguirre Baztán, 1995; Sanchiz Ochoa y Cantón Delgado, 1995; Heras Montoya, 1997; Nolla Cao, 1997; 
Parra Sabaj, 1998; Sanmartín Arce, 2000; Rivas y Calderón Almendros, 2002; Serra, 2004; Hammersley y Atkinson, 2005; Ortiz Cobo, 2006; Velasco y Díaz de Rada, 2006; Velasco, Díaz de Rada y García Castaño, 2006; Álvarez Álvarez, 2008a, 2008b; Jociles y Franze, 2008; Rockwell, 2001, 2009). Serra (2004: 166) considera que "la etnografía escolar o de la educación surge simplemente como consecuencia de haber seleccionado un campo determinado para realizar la etnografía”. Pero, podemos preguntarnos ¿qué es una etnografía?

Aguirre Baztán (1995:3), analizando el término etimológicamente, entiende que "la etnografía es el estudio descriptivo ("graphos") de la cultura ("ethnos") de una comunidad”. De este modo la etnografía escolar se ocuparía de realizar estudios descriptivos de la cultura escolar, tanto a nivel de los centros como de las aulas. Velasco y Díaz de Rada (2006: 10) consideran que "la etnografía de la escuela no es más que el resultado de aplicar una práctica etnográfica y una reflexión antropológica al estudio de la institución escolar”. Su principal característica es que el etnógrafo participa, abiertamente o de manera encubierta, en la vida diaria de las personas durante un periodo de tiempo, observando qué sucede, escuchando qué se dice, haciendo preguntas; de hecho, haciendo acopio de cualquier dato disponible que pueda arrojar un poco de luz sobre el tema en que se centra la investigación (Hammersley y Atkinson, 2005: 15).

Serra (2004: 165) lo expresa perfectamente: "el término etnografía se refiere al trabajo, el proceso o la forma de investigación que nos permite realizar un estudio descriptivo y un análisis teóricamente orientado de una cultura o de algunos aspectos concretos de una cultura, y, por otra, al resultado final de este trabajo (la monografía o el texto que contiene la descripción de la cultura en cuestión)".

\section{2. ¿QUÉ CARACTERIZA LA ETNOGRAFÍA ESCOLAR?}

Goetz y Le Compte (1988: 28-29) caracterizan la etnografía escolar a través de tres notas fundamentales:

1. Las estrategias utilizadas proporcionan datos fenomenológicos; éstos representan la concepción del mundo de los participantes que están siendo investigados.

2. Segundo, las estrategias etnográficas de investigación empíricas y naturalistas. Se recurre a la observación participante y no participante para obtener datos empíricos de primera mano.

3. Tercero, la investigación etnográfica tiene un carácter holista. Pretende construir descripciones de fenómenos globales en sus diversos contextos y determinar, a partir de ellas, las complejas conexiones de causas y consecuencias que afectan el comportamiento y las creencias en relación con dichos fenómenos.

En etnografía escolar es fundamental la participación prolongada del investigador en el contexto a estudiar estudiando el punto de vista de "los nativos". La participación prolongada permite crear relaciones cercanas que favorecen la recogida de unos datos fiables que, de otro modo, serían muy difíciles de lograr y de comprender. El etnógrafo necesita convivir con el grupo a estudiar durante periodos de tiempo continuados para comprender las interacciones que se producen entre sus miembros y poder dar cuenta 
fiel de las dialécticas relaciones que se producen entre las interacciones sociales y los significados que se construyen. Serra (2004: 167-168) plantea que:

Se considera que uno de los requisitos para la obtención de una buena etnografía es un trabajo de campo prolongado en el que se produzcan un contacto directo y una toma de datos sobre el terreno. La presencia en el campo y la vinculación con las personas que son objeto de estudio durante un periodo largo se consideran necesarias porque permiten reunir, en su ambiente natural, datos sobre el comportamiento de las personas y los acontecimientos, y situarlos en el contexto en el que adquieren significación, lo que facilita su comprensión y la formulación de hipótesis pertinentes.

Por otro lado, es precisa la observación participante del investigador como "nativo marginal" (Freilich, 1970). Esto hace referencia al hecho de que el etnógrafo nunca es uno más en el grupo que estudia, pero tiene que intentar integrarse lo más posible para realizar adecuadamente su investigación. Como plantean Hammersley y Atkinson (2005: 130), el etnógrafo tiene que "vivir en dos mundos simultáneamente, el de la participación y el de la investigación”. El mundo de la participación implica tratar de "ser uno más" en el grupo, procurando interferir lo menos posible en el modo de vida de los sujetos estudiados. El mundo de la investigación supone, además de trabajo de despacho, no olvidarse de que uno es investigador y tiene que asumir cierta distancia con aquello que observa. Por ello, se habla de que el etnógrafo asume una identidad de "nativo marginal".

En etnografía escolar también es importante la descripción reflexiva con carácter holista, lo cual supone para el etnógrafo la necesidad de realizar un trabajo en el que se relacione a los individuos, grupos u organizaciones con su entorno socio-económico, físico y simbólico de modo holístico.

El etnógrafo debe generar una "descripción densa" que aborde las principales estructuras de significación. Esta "descripción densa" no consiste en un relato pormenorizado de todas las observaciones realizadas, sino de aquellas que al investigador le resultan más significativas para dar a conocer la realidad que ha estudiado, es decir, aquellas que sirven para contextualizar lo más posible los casos estudiados y comprender sus dinámicas culturales. Serra (2004: 170) afirma que "hay que integrar el estudio de los problemas en el contexto general en el que se producen, y hemos de hacerlo guiados por los contextos teóricos que hemos seleccionado como adecuados”.

\section{3. ¿QUÉ PAPEL JUEGA EL INVESTIGADOR EN LA ETNOGRAFÍA ESCOLAR?}

El etnógrafo, para la gran mayoría de los autores, es el principal instrumento de investigación. De él dependen la selección de la temática a investigar, la filosofía que se adopte en el estudio, el acceso al campo, las relaciones con los sujetos estudiados, las observaciones e interpretaciones realizadas, y un largo etcétera. Como afirma Sanmartín Arce (2000: 139), el investigador es un reconstructor de la realidad, cuyo trabajo "exige paciencia y dedicación, atención esmerada y ferviente, fina observación y reflexión crítica de lo observado". Podemos preguntarnos cuáles son las principales exigencias que debe cumplir un investigador etnográfico en su quehacer cotidiano. Señalaré brevemente dos, que considero las más destacadas: el extrañamiento y el tratar de ser uno más. 
El extrañamiento fue señalado por primera vez por Malinowsky, en su clásico texto, Los argonautas del Pacífico Occidental, cuando apunta: "Imagínese que de repente está en tierra, rodeado de todos sus pertrechos, solo en una playa tropical cercana a un poblado indígena, mientras ve alejarse hasta desaparecer la lancha que le ha llevado hasta alli’" (Malinowsky, 1995: 24). El extrañamiento guarda una estrecha relación con dos aspectos básicos: (1) el desarraigo de abandonar los espacios que habitualmente uno frecuenta, y (2) el afrontamiento de una situación desconocida, que además ha de ser examinada para ser comprendida. Pero además, el extrañamiento, ha de ser una actitud vital en el etnógrafo a lo largo de todo el proceso de investigación, no únicamente en los primeros momentos. Esta actitud guarda una estrecha relación con la capacidad del investigador para afrontar con sensación de asombro cada observación realizada, por vulgar, familiar o rutinaria que parezca a simple vista, pues profundizando en su significado puede resultar muy reveladora para la investigación. Como afirma Rivas (2003: 111), las organizaciones escolares van más allá de estructuras y funciones, en ellas, "todo habla".

Un principio de procedimiento vinculado a esta cuestión lo aporta Barrio Maestre (1995: 165), cuando afirma que "el investigador tiene que poner en entredicho su etnocentrismo", es decir, el investigador tiene que juzgar la realidad desde el punto de vista de los miembros de la misma, mostrando capacidad para dejar a un lado sus concepciones previas. Para los investigadores en educación, al haber sido estudiantes algún día, todo resulta tan natural que es muy difícil extrañarse ante muchos aspectos prácticos, como plantea Delamont (1984: 163-164), “todos los que se ocupan de hacer investigación educacional han ido a la escuela y muchos de ellos han sido profesores, de ahí que la escena les resulte tan familiar".

Los antropólogos tienen dos términos que se emplean para designar el discurso de los miembros de la comunidad estudiada y el discurso propio acerca de la realidad cultural a investigar: emic y etic, respectivamente. El relato emic es el que se genera a partir de las opiniones y vivencias del observador cuando se convierte en un nativo más. El relato etic es el que permite ver la cultura con la mirada que realiza un observador externo a ella. La relación entre estos dos discursos es uno de los principales problemas a resolver porque entran en juego las cosmovisiones de los sujetos y las del investigador.

Pero el etnógrafo no sólo tiene que extrañarse con lo ajeno, sino que además tiene que tratar de integrarse parcialmente en el campo que estudia. Como plantea Barrio Maestre (1995: 164), "el etnógrafo tiene que familiarizarse con lo extraño y extrañarse de lo familiar". En este intento por ser uno más en la comunidad estudiada son fundamentales tres cualidades: intuición, reflexión y empatía (Heras Montoya, 1997: 16). El etnógrafo, en su viaje, tiene una brújula que le ayuda a ubicarse: en ocasiones la intuición le lleva a plantearse unas hipótesis, en otras ocasiones la reflexión le lleva a cuestionarse éstas o a construir otras y, otras veces, los afectos le permiten idear nuevas tentativas de explicación.

Estas cualidades personales son muy importantes y tienen que trabajarse permanentemente, en aras a conseguir una penetración estratégica dentro de la cultura a estudiar. Como plantea Woods (1987: 23), "así como se trabaja en el perfeccionamiento de un cuestionario, asi debe trabajarse en el desarrollo de las cualidades personales de curiosidad, penetración intuitiva, discreción, paciencia, decisión, vigor, memoria y el arte de escuchar y observar". 


\section{4. ¿CUÁLES SON LOS PRINCIPALES MOMENTOS EN UN ESTUDIO ETNOGRÁFICO?}

El proceso etnográfico no es un proceso lineal sino circular, aunque en él pueden identificarse diferentes momentos que en la práctica pueden darse, y de hecho se dan, simultáneamente. En este apartado señalo cuatro de estas etapas que considero fundamentales: la negociación y el acceso al campo, el trabajo de campo propiamente, el análisis de los datos y la elaboración del informe etnográfico.

\subsection{NEGOCIACIÓN Y ACCESO AL CAMPO}

El ingreso en el campo de estudio suele ser un problema al que aluden todas las obras sobre etnografía, y es que como muy acertadamente plantea Stake (2005: 58),

Casi siempre, la recogida de datos "se juega en casa" de alguien. En la mayoría de los casos, supone al menos una pequeña invasión de la vida privada. Los procedimientos para obtener respuesta se basan en que siempre se da por supuesta la necesidad de obtener permisos. ¿A quién corresponde el espacio en que nos movemos?

En la investigación educativa etnográfica es preciso negociar a diferentes niveles: dirección, administración, profesorado, alumnado, familias, etc., y se necesita la aprobación y/o consentimiento antes de iniciar el estudio empírico.

La fase de la negociación que nos abre o nos cierra las puertas al campo de estudio es obligada y puede determinar en buena medida el curso de la investigación, pues como Woods (1987: 37) mantiene, en esta fase "en el fondo, se trata de venderse a sí mismo como una persona digna de crédito que lleva a cabo un proyecto de valor". Se trata de una fase difícil porque en este momento están a flor de piel los intereses de todas las personas implicadas: investigadores, profesorado, dirección del centro, administración, etc. Cualquier pequeño indicio de que el estudio pueda perjudicar la imagen de alguien o de alguna institución puede ser determinante en el estudio que se desarrolle. En realidad, como plantean Sanchiz Ochoa y Cantón Delgado (1995: 129), la negociación, la entrada al campo y la recogida de información no son fases distintas porque "negociando el acceso nos hacemos con un tipo de información muy valiosa, y porque de algún modo esa negociación es un proceso permanente. Los primeros momentos son tan cruciales como el resto de los momentos, sólo que en el comienzo lo ignoramos casi todo".

Ahora bien, una vez negociada la entrada, el acceso al campo suele ser también conflictivo, pues entrar en un campo no significa permanecer en él en exclusiva. Acceder a un campo implica penetrar en las culturas grupales así como invadir determinados espacios que previamente eran habitados por otros. Como afirman Hammersley y Atkinson (1995: 72-73):

El acceso no es sólo una cuestión de presencia o ausencia física. Es mucho más que una simple cuestión de conseguir o poseer un permiso para llevar a cabo la investigación. (...) En muchos lugares, mientras la presencia física no representa en sí un problema, la actividad investigadora sí puede presentarlo. 


\subsection{TRABAJO DE CAMPO}

El trabajo de campo frecuentemente ha sido idealizado, así como la negociación y el acceso. Sanchiz Ochoa y Cantón Delgado (1995: 128) han escrito:

Si algo hay más idealizado que el trabajo de campo en la disciplina es el modo y manera en que ésta se inicia. Inicios idealizados por defecto, idealizados por silenciados: demasiada contingencia prosaica. Pero acaso ni lo uno ni lo otro, trabajo de campo y acceso-adaptación, merecen en justicia tanta solemnidad, atravesados como suelen estar de pesares y despropósitos.

La fase del trabajo de campo es una etapa de la investigación también problemática, pues como su propio nombre indica, se desarrolla en el espacio en el que habita el grupo a estudiar. En esta etapa básicamente se recoge la información con la que se trabajará posteriormente realizando los pertinentes análisis, aunque, como es evidente, ya muchos de estos análisis (reflexiones, interpretaciones, etc.) se van produciendo a la par de la recogida de datos. Es fundamental conseguir que la comunidad educativa deposite confianza en el investigador. Rockwell (2009: 55), considera que "la confianza se gana al no involucrarse directamente en los problemas particulares que ocurren entre las personas y, sobre todo, al no tomar ninguna acción que pudiera perjudicarlos”.

Las principales técnicas de recogida de información en etnografía son tres: la observación participante, la entrevista y el análisis documental. No obstante, en función del estudio pueden además emplearse otras, y conviene señalar que estas técnicas deben contribuir a un fin básico: la triangulación de perspectivas.

La observación participante es considerada la técnica por excelencia de la etnografía (Spradley, 1980). Ello es así porque "la observación participante se entiende como forma condensada, capaz de lograr la objetividad por medio de una observación próxima y sensible, y de captar a la vez los significados que dan los sujetos de estudio a su comportamiento. (...) La observación y la observación participante proporcionan descripciones, es decir, discurso propio del investigador" (Velasco y Díaz de Rada, 2006: 34). Para observar, lo ideal es modificar lo menos posible la situación objeto de estudio, adoptando un modo de actuar "de mosca en la pared", para observar las situaciones tal y como acontecen provocando la menor interferencia posible. Señala Woods (1987: 56), que "los principales requisitos de la observación son, naturalmente, un ojo avizor, un oído fino y una buena memoria”.

Por otra parte, los datos de la observación pueden recogerse con diferentes instrumentos: diarios de campo, registros anecdóticos, grabaciones, etc. Plantea Rockwell (2009: 59) que "una parte importante del trabajo de campo es el tiempo dedicado a escribir. La actividad de escribir nos coloca en una categoría rara, diferente de la de otras personas que frecuentan las escuelas. Casi no hay en ningún lugar la exhibición continua de comportamientos tan extraños como los que mostramos los etnógrafos".

La entrevista, por su parte, es la segunda estrategia fundamental en los estudios etnográficos. Su relevancia radica en que "tejida sobre el diálogo, proporciona discurso ajeno, de los sujetos de estudio" (Velasco y Díaz de Rada, 2006: 34). Me parecen especialmente significativas dos cuestiones apuntadas por estos autores: 
1. Tejida sobre el diálogo, es decir, evitando la formalidad siempre que sea posible, tendiendo a la conversación informal. Woods (1987: 82) plantea que "entrevista no es precisamente un término afortunado, pues implica una formalidad que el etnógrafo trata de evitar".

2. Proporciona discurso ajeno, es decir, los sujetos de estudio ofrecen su visión sobre los particulares que se comenten.

Las principales características que el etnógrafo debe tener para realizar entrevistas, según Woods (1987: 77), "giran siempre en torno a la confianza, la curiosidad y la naturalidad”. Otra cuestión es determinar quiénes serán los informantes principales del estudio, pues no siempre es una tarea sencilla, y en ocasiones es necesario realizar una tarea de "vagabundeo" (Goetz y Le Compte, 1988: 108). Stake (2005: 58) plantea que, " $a$ menos que se haya tenido una experiencia negativa reciente, las personas generalmente cooperan, les suele gustar que se conozca su historia, se sienten felices de ayudar a alguien a hacer su trabajo, aunque no sean optimistas sobre el beneficio que les pueda procurar la investigación”. La principal ventaja de esta técnica es que estimula el flujo de los datos y que ofrece una información personal que, de otro modo, sería imposible conocer. Los foros de discusión también constituyen, desde la etnografía, una forma particular de entrevista, aconsejable cuando el tema a tratar genera conflicto entre los miembros de la comunidad.

El análisis de documentos es otra técnica habitual en los estudios etnográficos, generalmente considerada como un apoyo a la observación. Básicamente consiste en un rastreo de materiales en formato papel, vídeo, audio, ya sean producidos por los miembros de la comunidad estudiada o por el propio investigador. San Fabián (1992: 35) muestra la enorme diversidad de documentos analizables: "cartas, memorias, autobiografías, periódicos, diarios, libros de texto, notas de suicidio, artículos, epitafios, casos, historias de vida, historias médicas, panfletos políticos, publicaciones oficiales, fotografías, películas, listas, registros, directorios, agendas", pues todos ellos, potencialmente, ofrecen una información variada que necesariamente hay que contemplar.

Dentro de esta variedad es posible trabajar con documentos oficiales y con documentos personales. Ambos tienen que examinarse teniendo en cuenta un aspecto fundamental: los documentos oficiales pueden ofrecer simplemente visiones "deseables" y los documentos personales pueden ofrecer simplemente visiones "autocomplacientes". En etnografía también es muy frecuente emplear alguna estrategia de grabación o fotografía porque permite "volver" sobre la realidad objeto de estudio. Estos materiales también constituyen informaciones privilegiadas que hay que analizar.

\subsection{ANÁLISIS DE DATOS}

La metodología etnográfica está caracterizada por el trabajo de campo, en el cual debe jugar un papel muy destacado la interpretación de los significados, el análisis de la estructura social y de los roles en la comunidad estudiada (Mauss, 1974; Woods, 1987; Goetz y Lecompte, 1988; Torres, 1988; Cohen y Manion, 1990; Martínez Rodríguez, 1990, Aguirre Baztán, 1995; Sanchiz Ochoa y Cantón Delgado, 1995; Heras Montoya, 1997; Serra, 2004; Hammersley y Atkinson, 2005; Ortiz Cobo, 2006; Álvarez Álvarez, 2008a, 2008b; Velasco y Díaz de Rada, 2006; Rockwell, 2001, 2009). 
El análisis de los datos es un aspecto sumamente delicado y complicado, pues el etnógrafo recoge gran cantidad de material de diversas fuentes, en diferentes soportes, y necesariamente debe hacer uso de él. Muchos son los autores que plantean que analizar los datos genera cierta angustia, al tener que elaborar un informe científico, en el que además se rechazan muchas ideas. Rodríguez Gómez, Gil Flores y García Jiménez (1996: 201) plantean que

El análisis de datos es visto por algunos como una de las tareas de mayor dificultad en el proceso de investigación cualitativa. El carácter polisémico de los datos, su naturaleza predominantemente verbal, su irrepetibilidad o el gran volumen de datos que suelen recogerse en el curso de la investigación, hacen que el análisis entrañe dificultad y complejidad.

¿En qué consiste este proceso? Stake (2005: 67) ha escrito: "El análisis significa esencialmente poner algo aparte. Ponemos aparte nuestras impresiones, nuestras observaciones. (...) Tenemos que separar la nueva impresión, y dar sentido a las partes. No al principio, la mitad y el final, no a esas partes, sino a aquéllas que son importantes para nosotros".

Para realizar este análisis, básicamente, se dan una serie de pasos, intrínsecamente ligados: una reflexión analítica sobre los datos, una selección, reducción y organización de los datos.

Reflexión analítica sobre los datos. A la par que el etnógrafo recoge los datos, realiza una tarea de reflexión que es fundamental para la organización y selección de los mismos. "Cuando se observa, se entrevista, se toman notas de campo y se confecciona el diario de investigación, la labor del etnógrafo no se limita a "registrar". También hay en ello reflexión, la que a su vez informa la serie de datos siguiente" (Woods, 1987: 135). El etnógrafo juega así un papel "centralizador" en todo el estudio. Su mente archiva y desecha, recoge y analiza, reflexiona sobre lo vivido, lo sentido, lo pensado, los datos recogidos. Sanchiz Ochoa y Cantón Delgado han afirmado:

Nadie niega ya el papel omnipresente de la subjetividad en el trabajo antropológico, ni la implicación del antropólogo en aquello que estudia. (...) Sabemos que los datos no se "recogen" tanto como se "construyen". Sabemos que después se interpretan. En verdad interpretamos desde el mismo momento en que iniciamos la "recogida de datos", y ciertamente ello da comienzo con el acceso al campo, o acaso antes (Sanchiz Ochoa y Cantón Delgado, 1995: 133).

Selección, reducción y organización de los datos. Dados los impresionantes volúmenes de información con los que trabaja un etnógrafo es preciso "apartar", como plantea Stake, aquello que es relevante para el estudio de aquello que no lo es tanto. Reducir los datos con los que se va a trabajar finalmente no es una tarea sencilla, pues implica dejar a un lado gran cantidad de material. ¿Cómo realizar esa selección? Los intereses del estudio tienen que guiar ese proceso. Hammersley y Atkinson (2005: 217) plantean que "las necesidades del etnógrafo, por supuesto, a la hora de decidir qué códigos son relevantes para los temas del trabajo en cuestión y para el análisis preliminar que acompaña a la recogida de información, son prioritarias". Una vez seleccionados los datos, es preciso organizar los mismos. Esta operación se puede realizar de modo "manual" o mediante el empleo de programas informáticos, pero, en cualquier caso, el etnógrafo tiene que tratar de ser consecuente con los intereses de su investigación, pues los programas informáticos, por ejemplo, hacen un excelente trabajo de recuento de frecuencias, pero no 
reflexionan sobre los significados que encierran las muestras que se someten al programa. Como afirma Rockwell (2009: 71), "si bien actualmente existen programas que facilitan el manejo de datos en la computadora, estos no eximen al investigador del necesario trabajo conceptual, previo a la codificación de los registros".

Categorización de los datos. En su esfuerzo por dar sentido a los datos recogidos, el etnógrafo genera y regenera categorías explicativas en las que agrupa los significados más relevantes recogidos. Esta categorización no es una tarea sencilla, ni definitiva, pues se encuentra sometida a permanente revisión y transformación, en cuanto aparece un nuevo dato que nos hace repensarla. Para Hammersley y Atkinson (2005: 213) "la identificación de categorías es un elemento central en el proceso de análisis”. Además, las categorías, con el objeto de llegar a formular unas conclusiones en el estudio, deben reagruparse formando redes que proporcionen información sobre las relaciones existentes entre las diferentes unidades de significado. Taylor y Bogdan (2000: 159) mencionan una serie de pasos que se deben dar en este proceso:

- Desarrollar categorías de codificación.

- Codificar todos los datos.

- Separar los datos pertenecientes a las diversas categorías de codificación.

- Ver qué datos han sobrado.

- Refinar el análisis.

Lejos de ser algo simple, la categorización exige tiempo, reflexión, paciencia, atención y esmero. Nada tienen que ver las primeras categorizaciones del investigador con las últimas. Mientras avanza en el proceso de análisis de los datos, éstos van virando: ampliándose, reduciéndose, agrupándose, reformulándose, etc.

A continuación, algunos autores como Taylor y Bogdan (2000: 171) destacan esta fase final en el análisis de los datos que denominan relativización de los datos y consiste en interpretar los datos categorizados en su contexto de recogida. Básicamente se trata de revisar el análisis a la luz del contexto de recogida de los datos para ver si realmente se está efectuando una categorización adecuada que refleja realmente el estudio hecho.

\subsection{ELABORACIÓN DEL INFORME ETNOGRÁFICO}

Elaborar el informe correspondiente al estudio realizado suele ser una tarea complicada. Spradley, (1980: 34), considera que "this last major task in the research cycle occurs toward the end of research project (...). Writing an etnography forces the investigator into a new more intensive kind of analysis". Tras horas y horas de lecturas, observaciones, conversaciones, entrevistas, análisis de documentos, etc. no es sencillo escribir, pues escribir significa divulgar, entre otras cosas. Stake (2005: 69) afirma que "la página no se escribe sola, sino cuando se descubre, y se somete a análisis, el ambiente adecuado, el momento adecuado, mediante la lectura repetida de las notas, con la reflexión profunda, para que después se revele el sentido y se nos escriba la hoja”.

Componer un informe de investigación implica precisar una estructura, adoptar un estilo de redacción, hacer esquemas, borradores y bosquejos y revisar una y otra vez lo producido, dándoselo a leer a otros siempre que sea posible, para comprobar que las personas ajenas al estudio lo comprenden. Escribir no es un acto mecánico y rutinario, 
sino un proceso creativo, reflexivo y personal. La redacción académica es una actividad intelectual dura, rigurosamente disciplinada, que exige dedicación, tranquilidad, optimismo y reflexión permanente. Como afirman Hammersley y Atkinson (2005: 260), "el mundo no se divide a sí mismo en capítulos y subtítulos según nuestra conveniencia”. La elaboración del informe etnográfico es una fase intensa, que precisa mucho tiempo, aunque no siempre es posible dedicarle todo el deseado. Como plantea Woods (1987: 185): "si la paz es esencial, también la prisa". Los informes suelen tener unos plazos y, aunque éstos tengan que ser reflexivos, en ocasiones, el tiempo se echa encima.

\section{5. ¿CÓMO CONSEGUIR QUE LA INVESTIGACIÓN ETNOGRÁFICA SEA VÁLIDA?}

La credibilidad de la investigación etnográfica ha sido una de las grandes preocupaciones en el contexto de la investigación educativa general. Frecuentemente se ha tildado a ésta de imprecisa, subjetivista, distorsionada, etc. Para garantizar la credibilidad de los datos etnográficos hay varias estrategias que el investigador tiene que conocer y emplear, que contribuyen a la validación de los mismos (Woods, 1987; Goetz y Lecompte, 1988; Aguirre Baztán, 1995; Heras Montoya, 1997; Serra, 2004; Hammersley y Atkinson, 2005; Álvarez Álvarez, 2008a, 2008b; Velasco y Díaz de Rada, 2006; Rockwell, 2001, 2009). Las principales son la contextualización, la saturación, la negociación con los implicados y la triangulación.

“Contextualizar" supone dar una visión panorámica de alguna cuestión. En etnografía, quedarse con una visión general de los hechos estudiados es reduccionista. Por ello, se plantea que no es conveniente examinar separadamente el entorno social y cultural del análisis de los mismos.

Suministrar contexto es ir mostrando las reglas que siguen los agentes de un modo de vida particular, proponiendo progresivamente nuevas ampliaciones ante sucesivas excepciones. Suministrar contexto también es dar la oportunidad al lector de la etnografía de ponerse en el lugar de aquéllos que viven una forma extraña de experiencia, ofreciéndole, de una manera ordenada, la mayor cantidad posible de claves significativas sobre su realidad concreta" (Velasco y Díaz de Rada, 2006: 236-237).

La saturación guarda relación con la justificación de una afirmación apoyándose en múltiples pruebas. Si sobre un tópico se desea indagar en especial puede que sea preciso observarlo, preguntar a los informantes más adecuados sobre él, analizar los documentos que se han generado sobre el mismo, incluso puede que sea necesario repetir estas estrategias, con el objeto de "saturar", de agotar las estrategias de búsqueda sobre el mismo, tratando de ver si los resultados obtenidos se mantienen en el tiempo.

La negociación con los implicados guarda relación con el encuentro entre los fines, los métodos y los resultados del etnógrafo y la opinión de los implicados, con el objeto de saber si hay acuerdo entre ellos, especialmente en lo que se refiere a los resultados que muestra el informe final. Para tal cuestión pueden organizarse foros, reuniones, etc. en los que se discutan cuestiones vinculadas a las diferentes fases del estudio o en las que se revisen escritos previos a la difusión del informe. González Riaño (1994: 217) afirma que "cuando las personas que intervienen en el control de la información la 
aceptan como justa, relevante y precisa, de algún modo están asegurando la validez o credibilidad de la misma".

La triangulación es la estrategia de validación de los datos más empleada y más conocida por los investigadores sociales. Básicamente puede entenderse como la puesta en relación de las aportaciones que realizan los diferentes agentes implicados en la investigación, incluido el punto de vista del investigador. Arias Valencia (2000: 8) plantea que "la principal meta de la triangulación es controlar el sesgo personal de los investigadores y cubrir las deficiencias intrínsecas de un investigador singular o una teoría única, o un mismo método de estudio y así incrementar la validez de los resultados”. Generalmente se habla de cuatro tipos de triangulación:

1. Triangulación de métodos: el investigador contrasta la información obtenida a través de una técnica (por ejemplo, la entrevista) con otras (la observación, la revisión documental...)

2. Triangulación de sujetos: el investigador trata de contrastar los puntos de vista de los miembros de la comunidad estudiada.

3. Triangulación de espacios y tiempos: se trata de aplicar las técnicas de recogida de información (observación, entrevista y análisis documental) en diferentes espacios y tiempos, para ver si los resultados obtenidos son consistentes.

4. Triangulación de expertos: se trata de que diferentes investigadores que se encuentren presentes en el campo a estudiar pongan en común sus visiones sobre el/los tema/s objeto de estudio.

\section{6. ¿QUÉ AVANCE SUPONE LA ETNOGRAFÍA ESCOLAR CON RESPECTO A OTROS MÉTODOS DE INVESTIGACIÓN EDUCATIVA?}

El principal aporte que hace la etnografía escolar reside en su capacidad para ilustrar al investigador sobre la escuela, permitiéndole comprender las dinámicas cotidianas escolares. Rockwell (2009: 30) considera que "la transformación más importante que logra la etnografía ocurre en quienes la practicamos. La experiencia de campo y el trabajo analítico deben cambiar la conciencia del investigador y modificar su manera de mirar los procesos educativos y sociales". Con demasiada frecuencia el investigador y el académico emplean métodos de investigación que no siempre garantizan el acercamiento y comprensión de la escuela con la profundidad que sería deseable (Rockwell, 2001; 2009). Sin embargo, la etnografía escolar permite el acercamiento y comprensión de la escuela, ofreciendo al investigador un modelo especialmente rico (Mauss, 1974; Fernández Enguita, 1985; Woods, 1987; Goetz y Lecompte, 1988; Torres, 1988; Martínez Rodríguez, 1990, San Fabián Maroto, 1992; Aguirre Baztán, 1995; Sanchiz Ochoa y Cantón Delgado, 1995; Heras Montoya, 1997; Nolla Cao, 1997; Parra Sabaj, 1998; Sanmartín Arce, 2000; Rivas y Calderón Almendros, 2002; Serra, 2004; Hammersley y Atkinson, 2005; Domínguez Figaredo, 2006; Ortiz Cobo, 2006; Velasco y Díaz de Rada, 2006; Velasco, Díaz de Rada y García Castaño, 2006; Álvarez Álvarez, 2008a, 2008b; Jociles y Franze, 2008; Rockwell, 2001, 2009).

La gran aportación de la etnografía radica en la técnica de la observación participante, porque permite acceder a un tipo de información que, si no, sería muy difícil de recoger, 
brindando al investigador la oportunidad de recoger dicha información en persona y conocer y vivir los procesos educativos en toda su complejidad.

A través de diversos estudios etnográficos, los etnógrafos han abierto campos de estudio, han ofrecido atentas descripciones, han aportado modelos para comprender la dinámica escolar y han explorado las perspectivas, estrategias y culturas de maestros y alumnos. (Woods, 1987; Goetz y Lecompte, 1988; Torres, 1988; Martínez Rodríguez, 1990, Aguirre Baztán, 1995; Sanchiz Ochoa y Cantón Delgado, 1995; Rivas y Calderón Almendros, 2002; Serra, 2004; Hammersley y Atkinson, 2005; Ortiz Cobo, 2006; Velasco y Díaz de Rada, 2006; Velasco, Díaz de Rada y García Castaño, 2006; Rockwell, 2001, 2009). Además, muchas de ellas han contribuido a mejorar la práctica de la enseñanza. Como plantea Torres (1988: 17), "las etnografías no deben quedarse exclusivamente en su dimensión descriptiva, sino que, como modalidad de investigación educativa que son, deben coadyuvar (...) una intervención pedagógica mejor”.

Ello es así porque la etnografía tiene varias finalidades íntimamente relacionadas: la descripción cultural, la interpretación de los datos para llegar a su comprensión, la difusión de los hallazgos, la mejora de la realidad educativa y la transformación del investigador (Woods, 1987; Goetz y Lecompte, 1988; Martínez Rodríguez, 1990, Aguirre Baztán, 1995; Sanchiz Ochoa y Cantón Delgado, 1995; Serra, 2004; Hammersley y Atkinson, 2005; Velasco y Díaz de Rada, 2006; Rockwell, 2001, 2009). Torres (1988: 14) afirma que:

Circunscribiéndonos al ámbito escolar, el objeto de la etnografía educativa se centra en descubrir lo que allí acontece cotidianamente a base de aportar datos significativos, de la forma más descriptiva posible, para luego interpretarlos y poder comprender e intervenir más adecuadamente en ese nicho ecológico que son las aulas.

\section{REFERENCIAS BIBLIOGRÁFICAS}

Aguirre Baztán, A. (Ed.) (1995). Etnografía. Metodología cualitativa en la investigación sociocultural. Barcelona: Marcombo.

Álvarez Álvarez, C. (2008a). "La etnografía como modelo de investigación educativa". Gazeta de Antropología, 24. (Disponible en Internet: http://www.ugr.es/ pwlac/).

Álvarez Álvarez, C. (2008b). Trabajo de investigación: "El diálogo como estrategia de educación en valores cívicos. Un estudio etnográfico en Primaria”. Director: José Luis San Fabián Maroto. Universidad de Oviedo. (Documento inédito).

Arias Valencia, M.M. (2000). "La triangulación metodológica: sus principios, alcances y limitaciones". Investigación y educación en enfermería, 1. (Disponible en Internet).

Barrio Maestre, J.M. (1995). "El aporte de las ciencias sociales a la Antropología de la Educación". Revista Complutense de Educación, 6, 159, 184.

Cohen, L. \& Manion, L. (1990). Métodos de investigación educativa. Madrid: La Muralla.

Delamont, S. (1984). La interacción didáctica. Madrid: Cincel.

Domínguez Figaredo, D. (2006). Etnografía educativa más allá de la etnografía escolar. Trasvase de significados al ciberespacio. En Forum Qualitative Social Research, 7.

Fernández Enguita, M. (1985). "Cualquier día, a cualquier hora: invitación a la etnografía de la escuela". Arbor, 477, 57-90.

Freilich, M. (1970). Marginal natives: Anthropologist at work. Nueva Cork: Harper \& Row.

Goetz, J.P. \& Lecompte, M.D. (1988). Etnografía y diseño cualitativo en investigación educativa. Madrid: Morata. 
González Riaño, X.A. (1994). Interferencia lingüística y escuela asturiana. Oviedo: Academia de la Llingua Asturiana.

Hammersley, M. y Atkinson, P. (2005). Etnografía. Métodos de investigación. Barcelona: Paidós.

Heras Montoya, L. (1997). Comprender el espacio educativo. Investigación etnográfica sobre un centro escolar. Málaga: Aljibe.

Jociles, M.I. \& Franze, A. (2008). ¿Es la escuela el problema? Madrid: Trotta.

Malinowski, B. (1995). Los argonautas del Pacífico Occidental. Barcelona: Península.

Martínez Rodríguez, J.B. (1990). Hacia un enfoque interpretativo de la enseñanza: etnografía y curriculum. Granada: Servicio de Publicaciones de la Universidad de Granada.

Mauss, M. (1974). Introducción a la etnografía. Madrid: Istmo.

Nolla cao, N. (1997). Etnografía: una alternativa más en la investigación pedagógica. Revista Cubana de Educación Media y Superior, 11, 107-115.

Ortiz Cobo, M. (2006). La mediación intercultural en contextos escolares: reflexiones acerca de una etnografía escolar. En Revista de Educación, 339, 563-594.

Parra Sabaj, M.E. (1998). La etnografía de la educación. En Cinta de Moebio, 3. en http://www. moebio.uchile.cl/03/frprin04.htm). [Consulta: de agosto de 2007].

Rivas, J.I. (2003). La perspectiva cultural de la organización escolar: marco institucional y comportamiento individual. En Educar, 31, 109-119.

Rivas, J.I. \& Calderón Almendros, I. (2002). La escuela desde la experiencia de los alumnos. Biografías y experiencia escolar. En X Simposium Interamericano de Investigación Etnográfica y cualitativa en Educación.

Rockwell, E. (2009). La experiencia etnográfica. Historia y cultura en los procesos educativos. Buenos Aires: Paidós.

Rockwell, E. (2001). La escuela cotidiana. México: FCE.

Rodríguez Gómez, G., Gil Flores, J. \& García Jiménez, E. (1996). Metodología de la investigación cualitativa. Málaga: Aljibe.

San Fabián Maroto, J.L. (1992). Evaluación etnográfica de la educación. En Blasco Sánchez, B., Cabo Martínez, M.R., San Fabián Maroto, J.L. \& Santiago Martínez, P. Perspectivas en la evaluación del sistema educativo. Departamento de Ciencias de la Educación de la Universidad de Oviedo. 13-53.

Sanchiz Ochoa, P. \& Cantón Delgado, M. (1995). Acceso y adaptación al campo. En Aguirre Baztán, A. (Ed.) Etnografía. Metodología cualitativa en la investigación sociocultural. Barcelona: Marcombo.

Sanmartín Arce, R. (2000). Etnografía de los valores. Revista Teoría de la educación, 12, 129-141.

Serra, C. (2004). Etnografía escolar, etnografía de la educación. Revista de Educación, 334, 165-176.

Spradley, J. (1980). Participant observation. Estados Unidos: Library of congress cataloging in publication data.

Stake, R.E. (2005). Investigación con estudio de casos. Madrid: Morata.

Stubbs, M. \& Delamont, S. (1978). Las relaciones profesor-alumno. Barcelona: Oikos-Tau.

Taylor, S.J. \& Bogdan, R. (2000). Introducción a los métodos cualitativos de investigación. La búsqueda de significados. Barcelona: Paidós.

Torres, J. (1988). La investigación etnográfica y la reconstrucción crítica en educación. En Goetz, J.P. \& Lecompte, M.D. Etnografía y diseño cualitativo en investigación educativa. Madrid: Morata.

Velasco, H. \& Díaz de Rada, Á. (2006). La lógica de la investigación etnográfica. Un modelo de trabajo para etnógrafos de escuela. Madrid: Trotta.

Velasco, H., Díaz de Rada, Á. \& García Castaño, F.J. (2006). Lecturas de etnografía para educadores. Madrid: Trotta.

Woods, P. (1987). La escuela por dentro. La etnografía en la investigación educativa. Barcelona: Paidós-MEC. 
